\title{
Imaging the gastrointestinal tract
}

\section{in 2008}

Charlotte Robinson, fellow in abdominal imaging, Vancouver General Hospital;

Shonit Punwani, consultant radiologist and Stuart Taylor, reader in clinical radiology and honorary consultant radiologist

Department of Specialist Imaging, University College Hospital, London

\section{Introduction}

Imaging of the gastrointestinal (GI) tract is evolving due to the advent of new technologies. The plain radiograph continues to provide a first-line investigation in the acute setting and is useful in the initial assessment of a variety of important diagnoses including bowel perforation, obstruction, volvulus and colitis. Where detailed luminal evaluation is required, fluoroscopic barium or water soluble single and double-contrast studies have to date been the investigations of choice. These studies can assess early mucosal changes, bowel transit time, discriminate between functional ileus and obstruction, and diagnose the cause of an obstruction. However, recent rapid advances in both computed tomography (CT) and magnetic resonance (MR) hardware, combined with their gradual increase in availability in the UK, are leading towards the replacement of fluoroscopic imaging for specific clinical indications. Leading the way in this trend is the progressive abandonment of the double-contrast barium enema in preference of computed tomographic colonography (CTC) which enables the entire lumen of the large bowel and the extra colonic organs to be visualised in one dataset with a higher sensitivity for cancer detection and an increased patient acceptability than barium enema. ${ }^{1} \mathrm{MR}$ imaging provides an additional advantage over $\mathrm{CT}$ as ionising radiation is not used in the generation of MR images and provides superior soft tissue contrast. MR is being increasingly advocated for the investigation of the small and large bowel. ${ }^{2}$
This article reviews the current role of contrast studies, highlights the development and establishment of CTC and reviews the recent advances in MR for luminal GI imaging.

\section{Contrast studies}

\section{Upper gastrointestinal tract}

Barium swallow studies remain the main investigation of dysphagia, allowing direct evaluation and inspection of the oesophageal mucosa and gastrooesophageal junction; an objective measurement of oesophageal contractibility, assessment of reflux, and the presence of strictures, pouches, hiatal herniae. ${ }^{3} \mathrm{~A}$ typical study lasts approximately 10 to 15 minutes and involves the patient swallowing barium during fluoroscopic screening and rapid frame rate radiographic exposures.

A barium meal (often performed together with a swallow) is required for the visualisation of the gastric mucosa and duodenum. During this investigation the patient ingests barium and carbex (effervescent granules) to provide a double contrast (gas and barium) view of the stomach. It is largely being superseded by endoscopy which allows direct visualisation and biopsy, coupled with no radiation dose. ${ }^{4}$

The small intestine is the most difficult part of the GI tract to evaluate due to its length and complex loops. Most endoscopic techniques are therefore limited to the examination of proximal or distal segments. Barium follow-through remains (in most centres) the test of choice for the investigation of abdominal pain, diarrhoea and in particular diseases manifesting mucosal abnormalities such as coeliac and Crohn's disease. ${ }^{5}$ The patient drinks approximately $500 \mathrm{ml}$ of barium diluted with water and liquid metoclopramide. Images are acquired approximately every 20 minutes to include the stomach, duodenum, jejunum and the proximal and terminal ileums. Subtle mucosal abnormalities are often detected (particularly of the terminal ileum in patients with Crohn's disease $)^{6}$; fistulous tracts, adhesions and more rarely intraluminal lesions are all demonstrable. Functional information about transit time and peristalsis can also be ascertained. The entire examination takes from one to two hours or longer, depending on the transit time of the ingested barium to the caecum.

Water soluble oral contrast studies are generally performed in the immediate postoperative period to assess anastomotic integrity (after surgery such as fundoplication or gastric bypass). The preference over barium is a consequence of the potential for free intra-abdominal barium to induce peritonitis. ${ }^{7}$

\section{Lower gastrointestinal tract}

Double-contrast barium enema is still performed in the majority of UK institutions, although there is a move towards CTC in an increasing number of centres, subject to resource availability. Double-contrast enema is used to investigate symptoms of lower GI malignancy such as a change in bowel habit, anaemia and unexplained weight loss. The patient ingests a low residue diet for 48 hours prior to the examination in combination with a cathartic bowel preparation. Barium is instilled through a rectal tube to coat the colonic mucosa, followed by gas to distend the colon. With adequate coating and distension, the entire colon can be visualised in exquisite detail on a series of abdominal and spot radiographs (Fig 1). The radiation dose of a typical examination, however, is usually less than an abdominal CT. Patient acceptability can be low and technically adequate (diagnostic) images can be difficult to obtain in the elderly and frail. If potentially malignant pathology is identified, the patient will require a colonoscopy (for biopsy) and a chest/abdomen/pelvis CT for staging.

Single contrast enemas are performed using a water soluble contrast such as gastrograffin to check for bowel anastomotic integrity. 


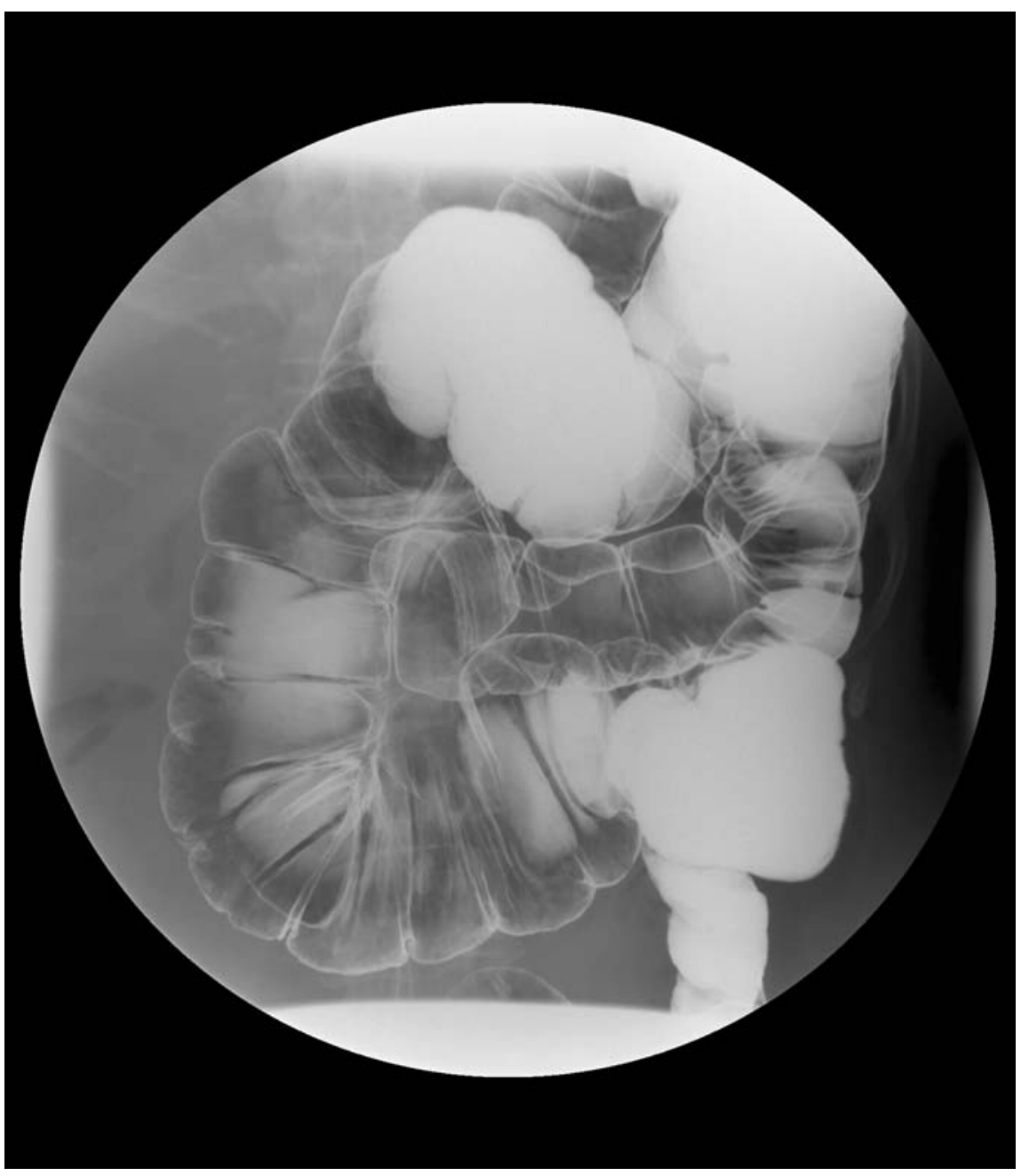

Fig 1. Part of a normal barium enema with sigmoid diverticular change.

\section{Key Points}

The plain abdominal $\mathrm{X}$-ray is an important first-line investigation for some suspected acute gastrointestinal disorders, notably perforation and bowel obstruction

Although barium studies continue to be the mainstay of luminal imaging, they are in part being replaced by new cross-sectional techniques

Computed tomographic colonography is evolving as a superior alternative to barium enema for imaging the colon

Improved magnetic resonance technology, coupled with its non-use of ionising radiation, has markedly increased its role in imaging of the small and large bowel, particularly in the young population with chronic disease

Ultrasound is a readily available imaging modality which is useful in depicting the terminal ileum, appendix and collections

KEY WORDS: barium, computed tomographic colonography, magnetic resonance imaging, plain abdominal X-ray, ultrasound

\section{Computed tomographic colonography}

Computed tomographic colonography involves full bowel preparation (akin to the double-contrast barium studies) followed by rectal gas insufflation and helical CT examination of the distended colon in the supine and prone positions with intravenous (iv) contrast. The resulting image dataset is then displayed on a workstation with complex image analysis software that renders images that simulate those obtained at conventional colonoscopy, hence the alternative term 'virtual colonoscopy.' Accurate diagnosis on CTC relies on technically good studies, the main aim of which is adequate bowel cleansing and distension (which can be difficult to obtain in the elderly and frail ${ }^{9}$ ). Clinical results suggest that the sensitivity of CTC in the detection of colorectal polyps and cancer exceeds that of a barium enema examination and approaches that of colonoscopy for medium (90\%) and large (95\%) polyps. ${ }^{10}$ As a result, the technique has disseminated rapidly into healthcare systems, notably in the USA, where it is advocated as a safer and more patientfriendly alternative to colonoscopy in colorectal cancer screening and detection of premalignant adenomatous polyps.

In the UK, CTC, although originally limited to specialist centres, is becoming increasingly disseminated throughout the NHS. Patient selection is mainly governed by clinician preference, although radiology departments are increasingly producing referral guidelines to optimise implementation of this new technology. Its main role lies in the investigation of symptomatic patients as an alternative to barium enema and conventional colonoscopy. Although not part of the National Bowel Cancer Screening Programme, CTC is often used in those with positive faecal occult blood tests, who are unfit or unwilling to undergo colonoscopy. CTC has a well-defined role in cases where conventional colonoscopy has failed to visualise the entire colon.

CTC is widely regarded as a safer alternative to conventional colonoscopy where colonic perforation rates have been 
reported at $0.2 \%$, compared with $0.08 \%$ for CTC, ${ }^{11}$ however, it remains relatively contraindicated in patients with any acute colonic inflammatory process.

\section{Magnetic resonance imaging studies}

\section{Small bowel magnetic resonance imaging}

Conventional fluoroscopic studies provide limited information regarding extramural manifestations of diseases affecting the bowel and are coupled with a radiation burden to a predominantly young patient population. The diagnosis of Crohn's disease is established by combining clinical, imaging, endoscopic and histological features.

The role of MRI has been to provide adequate information on the extension and severity of intramural and extramural abnormalities, and is increasingly being used to assess small bowel Crohn's disease, providing excellent depiction of mural and extra-mural tissues. ${ }^{12}$ In particular, specific findings on MRI have been proposed as accurate markers of disease activity, for example mural signal characteristics and contrast enhancement patterns (Fig 2). ${ }^{13}$

Small bowel distension for MR studies is most commonly achieved by oral administration of locust bean gum (extracted from the seeds of the carob tree) or mannitol 40 minutes prior to scanning. The patient is imaged in the prone position and not moved during the examination. Initially, dynamic 'real time cine loops' of small bowel peristalsis are acquired and used to assess the functional obstruction caused by strictures. Following iv buscopan peristalsis is reduced and highquality anatomical images of the bowel wall can be obtained. Furthermore, iv gadolinium contrast can be administered providing information about mural enhancement. Image acquisition times are in the order of 20 to 30 minutes.

\section{Colon magnetic resonance imaging}

The management of colonic inflammatory bowel disease (IBD) and assessment

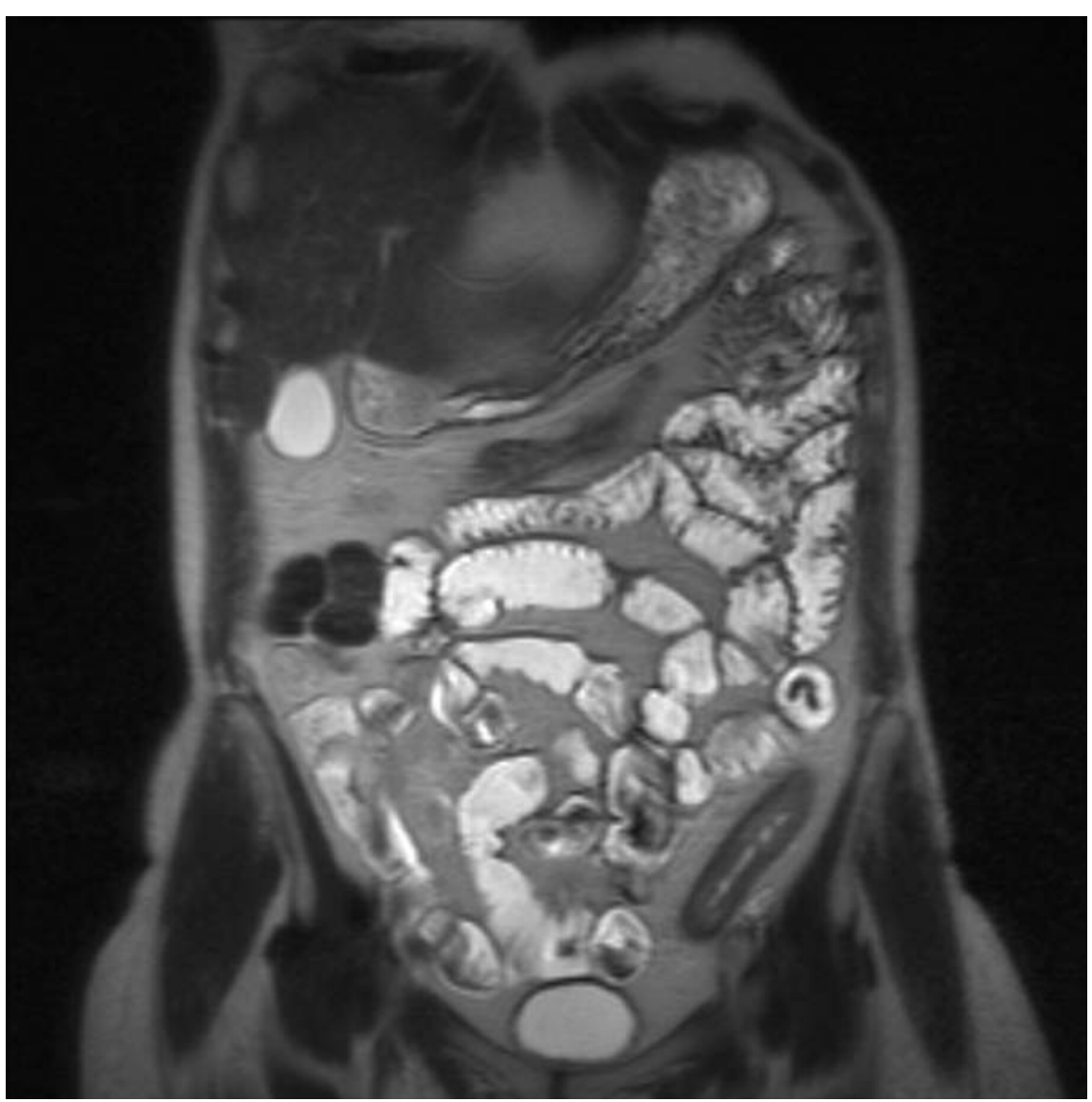

Fig 2. Magnetic resonance coronal T2 haste demonstrating thickening and enhancement of the distal descending colon in a patient with Crohn's disease.

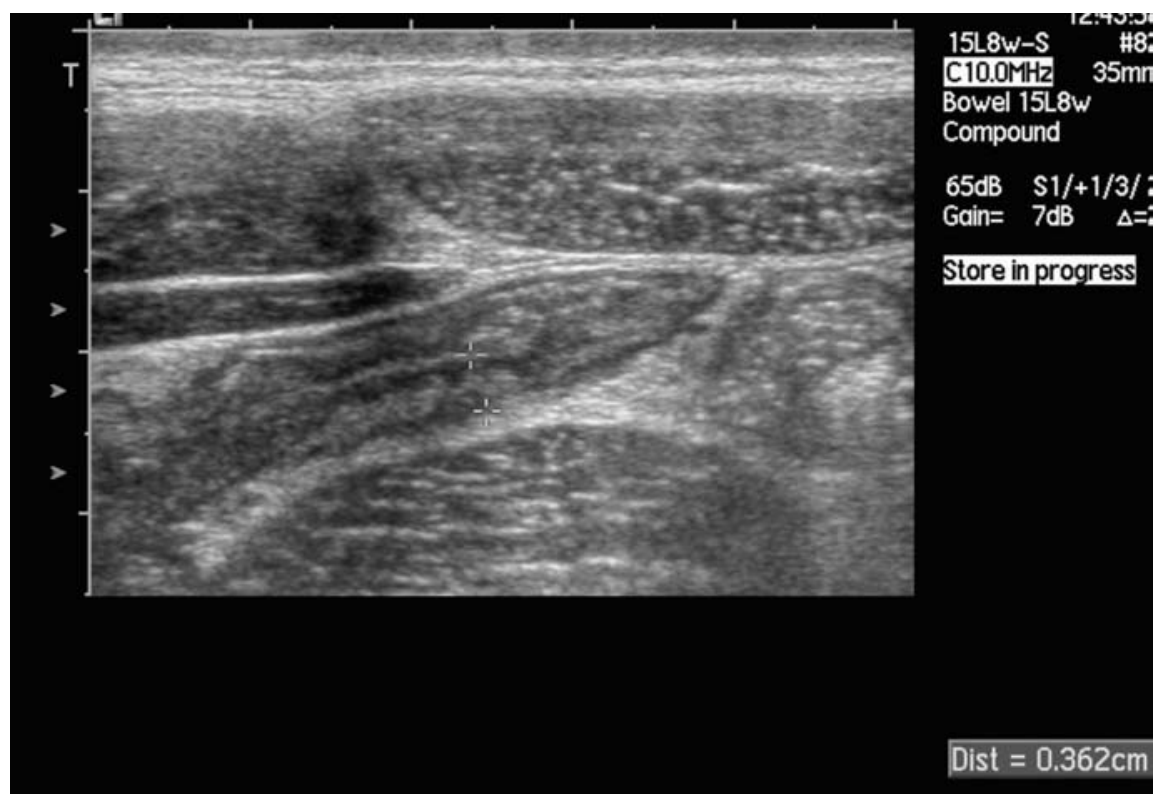

Fig 3. Mural terminal ileal thickening in a patient with Crohn's disease. 
of response to medication is aided by documenting the location, extent and the severity of the inflammation. Diagnostic endoscopy is considered a safe procedure but is invasive and often uncomfortable. MRI colonography has been advocated as a new and potentially useful investigation for assessment of those with IBD-related colitis with early studies of utility of MR colonography showing encouraging early results. ${ }^{14}$

\section{Pelvic magnetic resonance imaging}

Imaging patients with perianal disease with pelvic MRI provides anatomical information regarding fistulae and abscess which are of value to the surgeon prior to drainage and to the physician prior to biological therapy.

\section{Ultrasound}

As gas limits ultrasound, examination of the GI tract with this method has been limited. However, ultrasound is a relatively quick, often non-invasive investigation, with no radiation burden and a readily available resource, with good patient acceptability. Appropriate patient preparation with fasting prior to the ultrasound can improve image quality.

Small bowel ultrasound is used to identify and assess the terminal ileum, and up to approximately $15 \mathrm{~cm}$ of distal ileum, and is commonly used in the investigation of Crohn's disease. Mural thickness, local mesenteric inflammation and focal collections can be assessed (Fig 3). ${ }^{15}$ It can be useful where the diagnosis of appendicitis is clinically unclear. Endoscopic ultrasound is commonly used to assess upper GI malignancies, in particular oesophageal and pancreatic. Endoanal ultrasound is an established technique used for visualising the anal sphincter complex.

\section{Summary}

Plain radiographs remain useful in the acute setting. Barium studies continue to be the mainstay investigation of the GI tract but are gradually being superseded by newer techniques for example CTC and small bowel MRI. Further studies are underway to establish the clinical utility of MR and clarify its place in the repertoire of GI imaging studies.

\section{References}

1 Taylor SA, Laghi A, Lefere, P, Halligan S, Stoker J. ESGAR: consensus statement on CT colonography. Eur Radiol 2007;17:575-9.

2 Mackalski BA, Bernstein CN. New diagnostic imaging tools for inflammatory bowel disease. Gut 2006;55:733-41.

3 Furlow B. Barium swallow. Radiol Technol 2004;76:49-58.

4 Dooley CP, Larson AW, Stace NH et al. Double contrast barium meal and upper gastrointestinal endoscopy. A comparative study. Ann Intern Med 1984;101:538-45.

5 Planner AC, Phillips A, Bungay HK. The role of imaging in small bowel disease. Imaging 2006;18:228-56.

6 Halligan S, Nicholls S, Beattie RM et al. The role of small bowel radiology in the diagnosis and management of Crohn's disease. Acta Paediatr 1995;84:1375-8.

7 Grobmyer AJ, Kerlan RA, Peterson SM et al. Barium peritonitis. Am Surg 1984;50:116-20.
8 Halligan S, Fenlon HM. Virtual colonoscopy. BMJ 1999;319:1249-52.

9 Gryspeedt SS, Herman MJ, Baekelandt MA et al. Supine/left decubitus scanning: a valuable alternative to supine/prone scanning in CT colonography. Eur Radiol 2004;14:768-77.

10 Halligan S, Altman DG, Taylor SA et al. CT colonography in the detection of colorectal polyps and cancer: systematic review, metaanalysis, and proposed minimum data set for study level reporting. Radiology 2005;237:893-904.

11 Burling D, Halligan S, Slater A et al. Potentially serious adverse events at CT colonography in symptomatic patients; national survey of the United Kingdom. Radiology 2006;239:464-71.

12 Gourtsoyiannis N, Papanikolaou N, Karantanas A. Magnetic resonance imaging evaluation of small intestinal Crohn's disease. Best Pract Res Clin Gastroenterol 2006 Feb;20(1):137-56.

13 Gourtsoyiannis N, Papanikolaou N, Grammatikakis J et al. Assessment of Crohn's disease activity in the small bowel with MR and conventional enteroclysis: preliminary results. Eur Radiol 2004;14(6):1017-24.

14 Ajaj WM, Lauenstein TC, Pelster G et al. Magnetic resonance colonography for the detection of inflammatory diseases of the large bowel: quantifying the inflammatory activity. Gut 2005;54(2):257-63.

15 Sarrazin J, Wilson SR. Manifestations of Crohn disease at US. Radiographics 1996;16:499-520.

Address for correspondence: Dr S Taylor, Department of Specialist Imaging, Level 2 Podium, University College Hospital, 235 Euston Road, London NW1 2BU.

Email: stuart.taylor@uclh.nhs.uk 